\title{
Precision luminosity measurement of proton-proton collisions at the CMS experiment in Run 2
}

\author{
Peter Major* (on behalf of the CMS Collaboration) \\ Eötvös Loránd University, Budapest \\ MTA-ELTE Lendület Particle and Nuclear Physics Group \\ E-mail: pmajor@cern.ch ${ }^{\dagger}$
}

\begin{abstract}
Precision luminosity calibration is critical to determine fundamental parameters of the standard model and to constrain or to discover beyond-the-standard-model phenomena at LHC. The luminosity determination at the LHC interaction point 5 with the CMS detector, using proton-proton collisions at 13 and $5.02 \mathrm{TeV}$ during Run 2 of the LHC (2015-2018), is reported. The absolute luminosity scale is obtained using beam-separation ("van der Meer") scans. The dominant sources of systematic uncertainty are related to the knowledge of the scale of the beam separation provided by LHC magnets and the factorisability between the spatial components of the proton bunch density distributions in the transverse direction. When applying the van der Meer calibration to the entire data-taking period, a substantial contribution to the total uncertainty in the integrated luminosity originates from the measurement of the detector linearity and stability.
\end{abstract}

European Physical Society Conference on High Energy Physics - EPS-HEP2019 -

10-17 July, 2019

Ghent, Belgium

\footnotetext{
* Speaker.

$\dagger$ The author wishes to thank for their support the Hungarian Academy of Sciences "Lendület" (Momentum) Program (LP 2015-7/2015) and the National Research, Development and Innovation Office of Hungary (K 124845 and K 128713).
} 


\section{Introduction}

State of the art methods for measuring luminosity at hadron colliders makes use of the fact that the rate of any interaction scales linearly with the instantaneous luminosity. As a consequence the deposited energy or hit rate in selected detector modules can be used for luminosity determination. These selected modules are henceforth referred to as luminometers. At the CMS [1] experiment of LHC, five online (HFOC, HFET, PLT, BCM1F, and DT) and several offline luminometers (PCC, VTX, and RAMSES) are used [2, 3]. Absolute luminosity is obtained following the calibration of the luminometers using a van der Meer (vdM) method [4]. The resulting calibration constant is called $\sigma_{\mathrm{vis}}$. In this procedure several sources of systematic uncertainty need to be considered which, in this paper, are introduced separately for the calibration and the integration in Sections 2 and 3, respectively.

\section{Absolute luminosity calibration}

The background noise of the detectors is determined by super-separating the beams to $6 \sigma$ distance in both $x$ and $y$. The resulting rates are shown in Fig. 1 as measured by BCM1F. For accurate vdM fits the beam separation is a crucial parameter which is influenced by several effects. First the nominal beam position (position as set by the magnets) is calibrated using two different length scale scan methods that compare the observed luminous region and the nominal positions. A typical output is seen in Fig. 2. Then the separations are corrected for orbit drift (see Fig. 3). Finally the separations are compensated for the beam-beam deflection due to electromagnetic forces. The bunches not only deflect but exercise a focusing/defocusing effect on one another which is called the dynamic- $\beta$ effect. Both the deflection and the dynamic- $\beta$ effect are calculated in simulation and are handled by a correction on the separation and the rates respectively.

In the vdM method we assume factorisable bunch proton densities with respect to the $x$ and the $y$ directions. The bias and uncertainty due to this assumption is evaluated using the beamimaging and the offset scan methods [6]. Beam currents are important quantities for normalising the vdM scan results. They are affected by the presence of the ghost and satellite contents of the beam which are also taken into account. After all corrections are applied the consistency of the calibration constants derived from different bunches (Fig. 4) and scans are examined for a given detector, and the cross detector stability in the vdM fill is evaluated by comparing the measured luminosities by different detectors (Fig. 5).

\section{Integration and stability}

Luminometers age during data-taking periods due to the intense radiation. This results in a constant loss of efficiency which is monitored using short vdM scans, so called emittance scans [5], in the beginning and end of each fill. An example of the results is shown in Fig. 6. Based on this a correction is assigned to each luminometer. The rates in standard physics data-taking periods are much higher than during the $\mathrm{vdM}$ fills and hence the calibration needs to be extrapolated to these conditions. This requires a study of the linearity of the detectors. Figure 7 shows the calibration constant derived for PLT (in emittance scans) as a function of single bunch instantaneous 


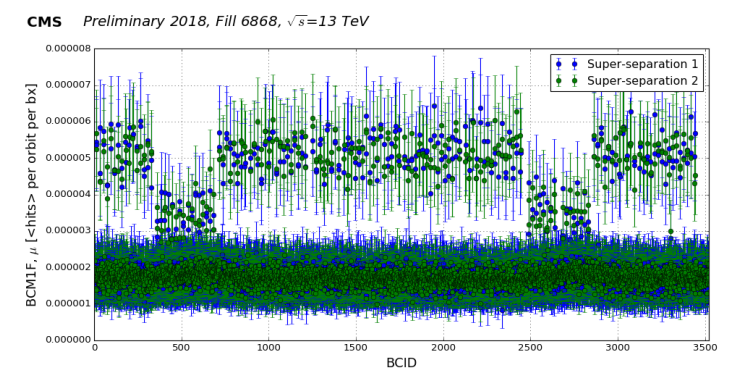

Figure 1: Rates measured by the BCM1F detector in the "super separation" scan. [2]

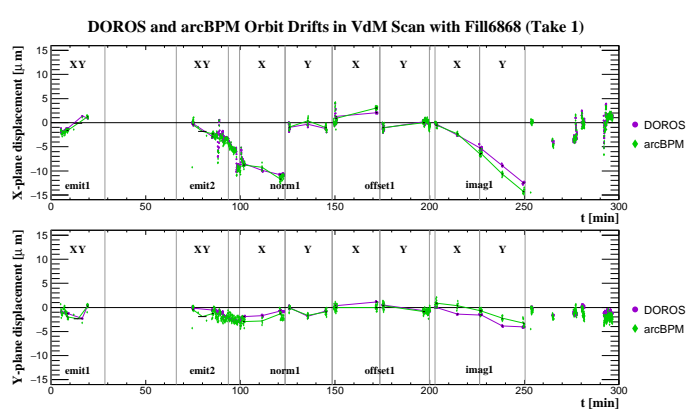

Figure 3: Orbit drift during the first part of the vdM scan in 2018. The $x$ drift is shown in the top plot and the $y$ in the bottom plot as measured by the DOROS and the arc beam position monitors. [2]

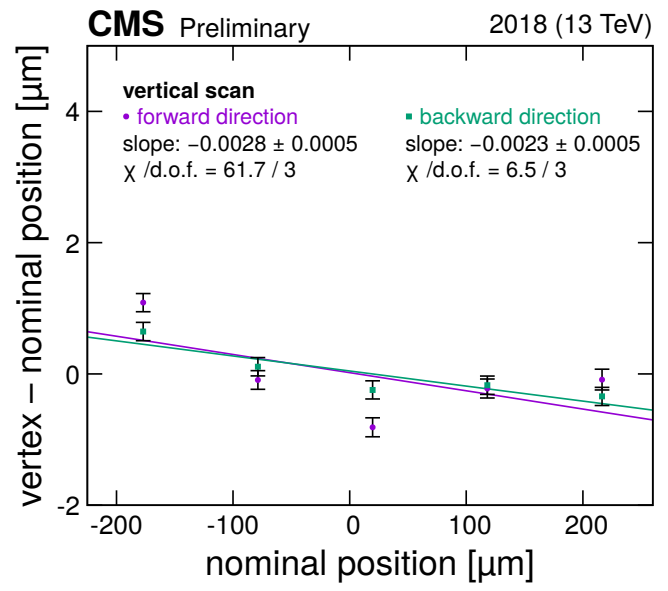

Figure 2: Difference between reconstructed luminous region position and nominal beam position, as a function of the latter. [2]

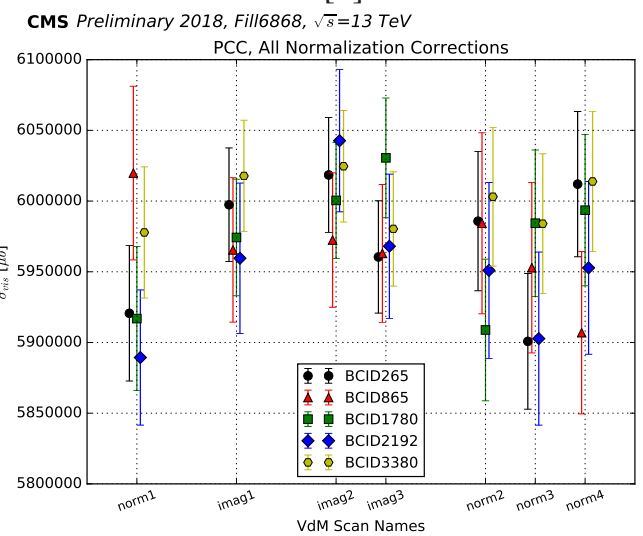

Figure 4: Visible cross section for the PCC for all vdM scans, for the five bunch crossings with data from the silicon pixel tracker available. [2]

luminosity (SBIL). We see that bunches leading the bunch train exhibit a different behaviour which is taken into account for the extrapolation and the corresponding uncertainty. After both linearity and efficiency corrections are applied and the two most stable detectors are chosen, their consistency is tested by examining the ratio of the measured luminosity as seen in Fig. 8. Plotting the ratio as a function of SBIL also reveals some residual relative nonlinearity. An example of the ratio of two luminometers as a function of SBIL is shown for HFOC and PLT in Fig. 9. Both effects are taken into account by assigning a corresponding uncertainty.

\section{Summary}

In this paper, I gave a brief overview of the sources of systematic uncertainty relevant to the luminosity determination procedure at CMS. The steps were illustrated using 2018 data as in Ref. [2]. Efforts are ongoing to improve and apply the presented techniques to all Run 2 (2015-2018) 


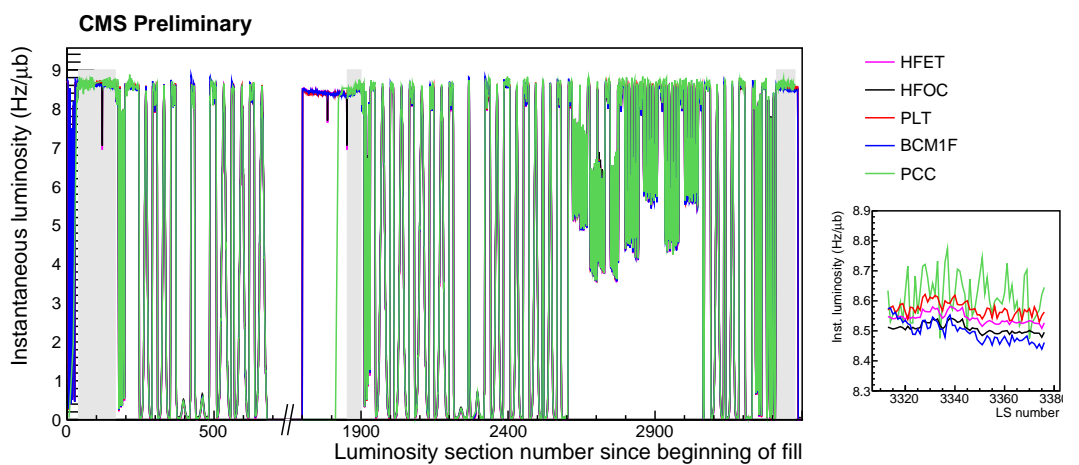

Figure 5: Instantaneous luminosity for the five independent luminometers during the vdM scan fill, showing the general agreement between all five luminometers. The inset shows a zoom in on one of the periods with stable luminosity, in which the slightly different absolute scales of the different luminometers can be observed. The three grey shaded rectangles indicate the periods used to calculate the total integrated luminosity for the cross-detector comparison. [2]

data to achieve a smaller total uncertainty for the whole data set. Table 1 shows a summary of the systematic uncertainties taken into account in 2018.

\begin{tabular}{|l|l|c|c|}
\hline & Systematic & Correction (\%) & Uncertainty (\%) \\
\hline \multirow{5}{*}{ Normalization } & Length scale & -0.8 & 0.2 \\
\cline { 2 - 4 } & Orbit drift & 0.2 & 0.1 \\
\cline { 2 - 4 } & $x-y$ factorisability & 0.0 & 2.0 \\
\cline { 2 - 4 } & Beam-beam deflection & 1.5 & \multirow{2}{*}{0.2} \\
\cline { 2 - 4 } & Dynamic- $\beta$ & -0.5 & 0.2 \\
\cline { 2 - 4 } & Beam current calibration & 2.3 & 0.1 \\
\cline { 2 - 4 } & Ghosts and satellites & 0.4 & 0.3 \\
\cline { 2 - 4 } & Scan to scan variation & - & 0.1 \\
\cline { 2 - 4 } & Bunch to bunch variation & - & 0.5 \\
\cline { 2 - 4 } & Cross-detector consistency & - & 0.1 \\
\cline { 2 - 4 } & Background subtraction & 0 to 0.8 & $0.1 \oplus 0.4$ \\
\hline \multirow{5}{*}{ Integration } & Afterglow (HFOC) & 0 to 4 & 0.6 \\
\cline { 2 - 4 } & Cross-detector stability & - & 1.1 \\
\cline { 2 - 4 } & Linearity & - & 2.5 \\
\cline { 2 - 4 } & CMS deadtime & - & $<.1$ \\
\hline & Total & & \\
\hline
\end{tabular}

Table 1: Summary of the sources of systematic uncertainty in the CMS luminosity measurement for $\sqrt{s}=13 \mathrm{TeV}$ proton-proton collisions in 2018. When applicable, the percentage correction is shown. [2]

\section{References}

[1] CMS Collaboration, The CMS experiment at the CERN LHC, JINST 3 (2008) S08004 [DOI:10.1088/1748-0221/3/08/S08004]

[2] CMS Collaboration, CMS luminosity measurement for the 2018 data-taking period at $\sqrt{s}=13 \mathrm{TeV}$, CMS-PAS-LUM-18-002 


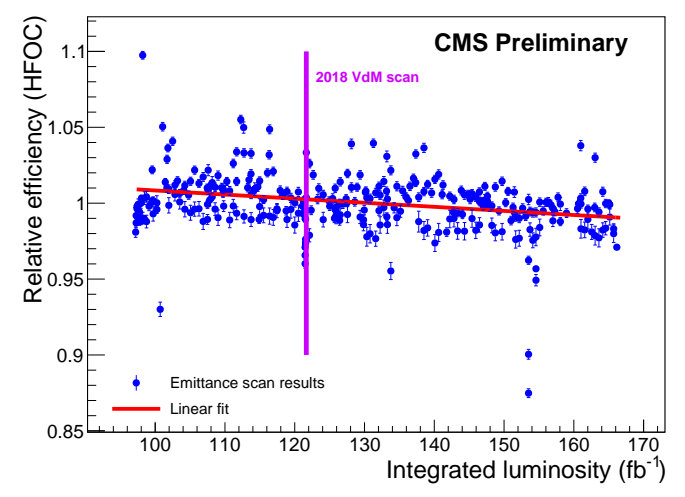

Figure 6: The efficiency of the HFOC detector relative to the efficiency at the $2018 \mathrm{vdM}$ scan. The observed decrease is due to the ageing of the HF. [2]

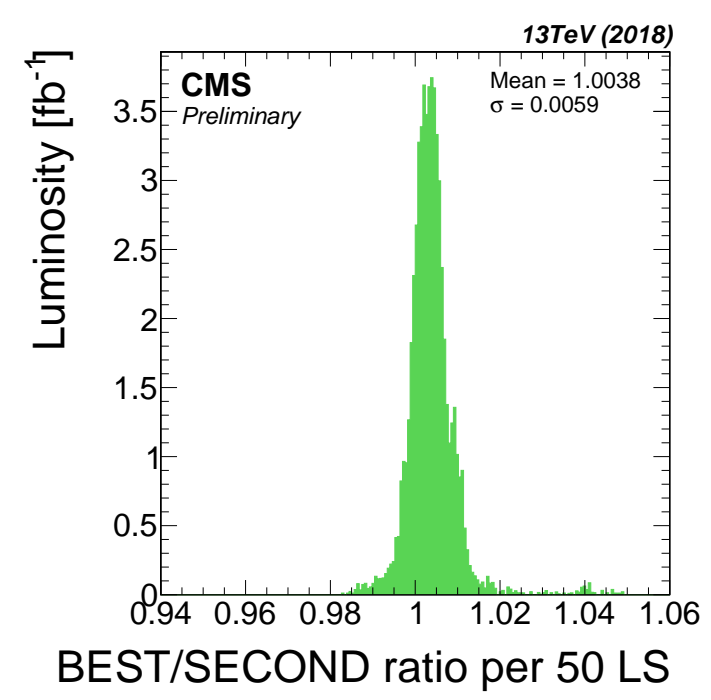

Figure 8: The ratio of the luminosity measured by the best and second-best luminometer. [2]

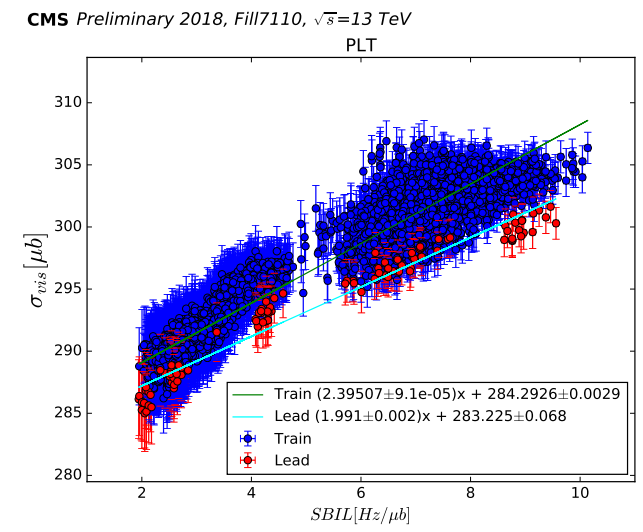

Figure 7: The calibration constant $\left(\sigma_{\mathrm{vis}}\right)$ calculated based on the emittance scans of fill 7110 separately for each bunch as the function of SBIL. Red points correspond to the bunches leading the bunch train. [7]

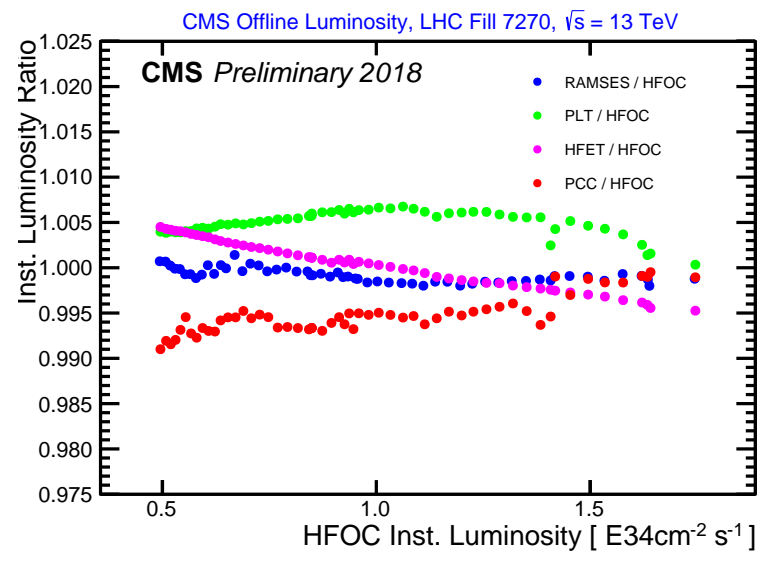

Figure 9: Luminosity ratios of different detector pairs as the function of instantaneous luminosity after all corrections are applied. The slope of the ratio is used to estimate the residual relative nonlinearity of the two detectors. [8]

[3] O. Karacheban on behalf of the CMS Collaboration, Performance of the BRIL luminometers at CMS for Run 2, in these proceedings

[4] S. van der Meer, Calibration of the effective beam height in the ISR, Technical Report CERN-ISR-PO-68-31, 1968.

[5] O. Karacheban on behalf of the CMS Collaboration, Emittance scans for CMS luminosity calibration in Run 2, in these proceedings

[6] P. Major on behalf of the CMS Collaboration, Studies of the factorisation of proton densities in van der Meer scans and its impact on precision luminosity measurements for CMS, in these proceedings

[7] CMS Collaboration, Long-term monitoring of delivered luminosity and calibration stability in CMS, CMS DP-2019/016, 017

[8] CMS Collaboration, Auxiliary material for the 2018 luminosity measurement, CMS DP-2019/024 